\begin{tabular}{|c|l|}
\hline Title & An improvement of level set equations via approximation of a distance function \\
\hline Author(s) & Hamamuki, Nao \\
\hline Citation & Hokkaido University Preprint Series in Mathematics, 1108, 1-16 \\
\hline Issue Date & 2018-02-02 \\
\hline DOI & 10.14943/81742 \\
\hline Doc URL & http://hdl.handle.net/2115/68267 \\
\hline Type & bulletin (article) \\
\hline Note & Mathematics Subject Classin cation 2010: 35D 40; 35F21; 35F 25 \\
\hline Information & document_20180202.pdf \\
\hline
\end{tabular}

Instructions for use 


\title{
An improvement of level set equations via approximation of a distance function
}

\author{
Nao Hamamuki *
}

February 2, 2018

\begin{abstract}
In the classical level set method, the slope of solutions can be very small or large, and it can make it difficult to get the precise level set numerically. In this paper, we introduce an improved level set equation whose solutions are close to the signed distance function to evolving interfaces. The improved equation is derived via approximation of the evolution equation for the distance function. Applying the comparison principle, we give an upper- and lower bound near the zero level set for the viscosity solution to the initial value problem.
\end{abstract}

Key words: level set method; improved level set equations; signed distance function; viscosity solutions

Mathematics Subject Classification 2010: 35D40; 35F21; 35F25

\section{Introduction}

Motivation. A level set method is a well-known technique to track a motion of an interface $\{\Gamma(t)\}_{t}$ in $\mathbf{R}^{n}$. The idea is that, given an initial interface $\Gamma(0)$, we represent it as the zero level set of some function $u_{0}(x)$, and then solve the initial value problem of a level set (partial differential) equation associated with a surface evolution equation for $\Gamma(t)$. Finally, we cut out the zero level of the solution $w(x, t)$ :

$$
\Gamma(t)=\left\{x \in \mathbf{R}^{n} \mid w(x, t)=0\right\}
$$

which is considered as the desired motion. Wide application of this method includes, e.g., the mean curvature flow equation originally developed by $[6,3]$. See also [8].

In this paper we study a level set equation given as the Hamilton-Jacobi equation:

$$
\partial_{t} w(x, t)+H(x, \nabla w(x, t))=0 \quad \text { in } \mathbf{R}^{n} \times(0, T)
$$

with the initial condition

$$
w(x, 0)=u_{0}(x) \in B U C\left(\mathbf{R}^{n}\right) \quad \text { in } \mathbf{R}^{n} .
$$

*Department of Mathematics, Hokkaido University, Kita 10, Nishi 8, Kita-Ku, Sapporo, Hokkaido, 0600810, Japan. E-mail: hnao@math.sci.hokudai.ac.jp 
Here $H$ is a continuous Hamiltonian, $\nabla w=\left(\partial_{x_{i}} w\right)_{i=1}^{n}$ denotes the gradient of $w$ with respect to $x$ and $B U C\left(\mathbf{R}^{n}\right)$ represents the set of bounded and uniformly continuous real-valued functions in $\mathbf{R}^{n}$.

Although mathematical formulation is great, in practice, it might become difficult to compute the precise zero level set when the slope of a solution $w$ near the zero level gets close to 0 or goes to $\infty$ as time develops. This can happen even if the initial slope is chosen suitably. To avoid such a bad (flat/steep) slope of solutions, one of famous methods is the reinitialization, which is especially useful for fluid flows $([17,16])$. The usual idea is that one stops the process of solving a level set equation after a little time and then modifies the solution at the stopping time so that the modified function approximates the signed distance function.

In this paper, however, we takes a different approach. We aim at keeping a good slope for a long time by changing the original level set equation. Namely, we solve the equation only once. We propose an improved level set equation of the form

$$
\partial_{t} u(x, t)+H(x, \nabla u(x, t))=u(x, t) G(x, \nabla u(x, t))
$$

with a new continuous function $G$. (Hereafter we use $w$ for the original equation (1.2) and $u$ for the improved equation (1.4).) Our goal is to demonstrate that, if we choose $G$ in a suitable way, the slope of the viscosity solution $u$ of (1.4) is close to one globally-in-time near the zero level set.

Another motivation of this work comes from [15]. There, an improved level set equation was proposed for the transport equation:

$$
\partial_{t} w(x, t)+\langle X(x), \nabla w(x, t)\rangle=0
$$

where $X: \mathbf{R}^{n} \rightarrow \mathbf{R}^{n}$ is a vector field and $\langle\cdot, \cdot\rangle$ denotes the standard inner product in $\mathbf{R}^{n}$. The improved equation proposed by Roisman is of the form

$$
\partial_{t} u(x, t)+\langle X(x), \nabla u(x, t)\rangle=u(x, t) \frac{\left\langle X^{\prime}(x) \nabla u(x, t), \nabla u(x, t)\right\rangle}{|\nabla u(x, t)|^{2}} .
$$

Here $X^{\prime}(x)$ stands for the Jacobian matrix of $X(x)$. This improved equation is derived so that, for a smooth solution $u$ of (1.4), the initial slope is preserved on the zero level along each flow determined by the vector field $X$. In other words, $G$ is chosen so that the derivative of $|\nabla u|^{2}$ is 0 along each flow. Our goals of this paper include justification and generalization of this method in a suitable sense. It turns out that, although our approach is different from [15], in the case of (1.5), our $G$ introduced later agrees with the function appearing on the right-hand side of (1.6) except in a small neighborhood of the singular point $|\nabla u|=0$.

Results. In order to introduce an improved level set equation, we focus on the equation for the signed distance function $d(x, t)$ to the interface, which is given by

$$
d(x, t)= \begin{cases}\operatorname{dist}(x, \Gamma(t)) & \text { if } x \in D^{+}(t), \\ 0 & \text { if } x \in \Gamma(t), \\ -\operatorname{dist}(x, \Gamma(t)) & \text { if } x \in D^{-}(t) .\end{cases}
$$

Here $\operatorname{dist}(x, \Gamma(t))=\inf \{|x-y| \mid y \in \Gamma(t)\}, w$ is the solution to (1.2) with (1.3) and $D^{ \pm}(t):=\left\{x \in \mathbf{R}^{n} \mid \pm w(x, t)>0\right\}$. The distance function is known to be a solution of 
the eikonal equation $|\nabla d(x, t)|=1$ both in the almost everywhere sense and in the viscosity sense. It is thus reasonable to use the signed distance function to guarantee that the slope remains one. Later we derive an evolution equation (3.1) for $d$, and then approximating it near the interface, we propose a suitable definition of $G$ in (1.4). It turns out that, if the initial data $u_{0}$ is equal to $d(\cdot, 0)$ near the initial interface $\Gamma(0)$ and if $d$ is smooth near $\Gamma(t)$, then for every $\varepsilon>0$,

$$
\left\{\begin{array}{ll}
e^{-\varepsilon t} d(x, t) \leqq u(x, t) \leqq e^{\varepsilon t} d(x, t) & \text { if } d(x, t) \geqq 0, \\
e^{\varepsilon t} d(x, t) \leqq u(x, t) \leqq e^{-\varepsilon t} d(x, t) & \text { if } d(x, t) \leqq 0
\end{array} \quad \text { near } \Gamma(t) .\right.
$$

This is a comparison between $u$ and slight modifications of $d$. In this sense, $u$ approximates $d$ and the slope of $u$ is kept to be one near $\Gamma(t)$. We also give a numerical result for this.

Our result (1.8) gives a kind of gradient estimates for the solution $u$ to (1.4) both from above and below. Although an upper gradient estimate (Lipschitz estimate) has been well studied for viscosity solutions (e.g., [2]), there is few result related to a lower gradient estimate. In [13] the author gives a lower gradient estimate for first order equations with a convex Hamiltonian. In our case, (1.4) is not convex even if the original $H$ in (1.2) is convex. For this reason, the same approach as [13] can not be applied. Recently, in [7] Fujita derived a lower gradient estimate globally-in-space both for second and first order equations.

Motivated by the reinitialization mentioned above, in a joint work with Ntovoris [11] we introduced another type of improved equations of the form

$$
\partial_{t} u^{\theta}(x, t)+H\left(x, \nabla u^{\theta}(x, t)\right)=\theta \beta\left(u^{\theta}(x, t)\right) h\left(\nabla u^{\theta}(x, t)\right) .
$$

Here $\theta>0$ is a parameter and $\beta: \mathbf{R} \rightarrow \mathbf{R}$ and $h: \mathbf{R}^{n} \rightarrow \mathbf{R}$ are typically chosen as a smooth approximation of the sign function such as $\beta(r)=r / \sqrt{r^{2}+\varepsilon^{2}}$ and $h(p)=1-|p|$, respectively. The equation (1.9) is obtained via the limit of the iterative procedure of solving original (1.2) and a so-called corrector equation to yield a distance function. Among other results, we proved in [11] that

$$
\lim _{\substack{(y, s, \theta) \rightarrow(x, t, \infty) \\ s \leqq t}} u^{\theta}(y, s)=d(x, t) \quad \text { for all }(x, t) \in \mathbf{R}^{n} \times(0, T) .
$$

In other words, $u^{\theta}$ converges to $d$ from below in time, Since a rate of convergence was not derived in [11], we give a partial answer in this paper. This also gives a comparison and an approximation of $d$ like (1.8).

Organization. Section 2 provides a brief summary of the theory of viscosity solutions and level set equations. In Section 3, after we explain how to choose $G$, we prove (1.8) for a solution $u$ of (1.4). Section 4 contains examples and numerical results. In Section 5 we derive a new estimate for a solution $u^{\theta}$ of (1.9).

This paper is not just a survey of [11] and [9, 10] ([9] is a dissertation and [10] is a Japanese article), where the result (1.8) has been stated, but also shows some progress on generalization and simplification of assumptions and discussions; (S2), (H2), (G1), Proposition 2.5 and Lemma 3.3 etc. are related. Moreover, numerical tests and an estimate for $u^{\theta}$ in (1.9) are new contents. 


\section{Preliminaries -Viscosity solutions and level set equations-}

This section gives a quick review of analytical tools needed in this paper. We first recall a notion of viscosity solutions ([4] and [8, Chapter 2, 3]) and a comparison result for them, which will play an important role in our study. We then describe basic facts of the level set equation (1.2) together with some results related to our improved equation (1.4), for which we refer to [8, Chapter 4] and [11].

We state a definition and a comparison result for (1.4). Clearly, those for (1.2) are included as the case $G \equiv 0$. We assume that $H, G: \mathbf{R}^{n} \times \mathbf{R}^{n} \rightarrow \mathbf{R}$ are continuous.

Definition 1 (Viscosity solution). We say that an upper semicontinuous (resp. lower semicontinuous) function $u: \mathbf{R}^{n} \times(0, T) \rightarrow \mathbf{R}$ is a viscosity subsolution (resp. viscosity supersolution) of (1.4) if

$$
\partial_{t} \phi(z, s)+H(z, \nabla \phi(z, s))-u(z, s) G(z, \nabla \phi(z, s)) \leqq 0(\text { resp. } \geqq 0)
$$

for all $(z, s) \in \mathbf{R}^{n} \times(0, T)$ and $\phi \in C^{1}\left(\mathbf{R}^{n} \times(0, T)\right)$ such that $u-\phi$ attains a local maximum (resp. local minimum) at $(z, s)$. Such a smooth function $\phi$ is called a test function for $u$ at $(z, s)$ from above (resp. below). If $u$ is both a viscosity sub- and supersolution, then it is called a viscosity solution.

To guarantee the comparison principle we assume

(CP) (i) For both $F=H$ and $G$, there exists a modulus $\omega$ (i.e., a nondecreasing function $\omega:[0, \infty) \rightarrow[0, \infty)$ satisfying $\left.\lim _{r \rightarrow 0} \omega(r)=\omega(0)=0\right)$ such that, for all $x, y, p, q \in \mathbf{R}^{n}$

$$
|F(x, p)-F(y, q)| \leqq \omega((1+|p|+|q|)|x-y|+|p-q|)
$$

(ii) $G$ is bounded in $\mathbf{R}^{n} \times \mathbf{R}^{n}$, i.e., $\|G\|:=\sup _{\mathbf{R}^{n} \times \mathbf{R}^{n}}|G|<\infty$.

Theorem 2.1 (Comparison principle [4, Section 8], [8, Chapter 3]). Let $u, v: \mathbf{R}^{n} \times[0, T) \rightarrow$ $\mathbf{R}$, and assume that $u$ and $-v$ are upper semicontinuous and bounded from above in $\mathbf{R}^{n} \times$ $[0, T)$. Assume that $u$ and $v$ are, respectively, a viscosity sub-and supersolution of (1.4). If $u(\cdot, 0) \leqq v(\cdot, 0)$ in $\mathbf{R}^{n}$, then $u \leqq v$ in $\mathbf{R}^{n} \times(0, T)$.

Throughout this paper we assume $(\mathrm{CP})$. As is well-known, the comparison principle implies uniqueness of viscosity solutions of the initial value problem.

Existence of viscosity solutions is shown by Perron's method. In this paper we omit it; see $[4$, Section 4] and [8, Section 2.4] for the detail. The unique viscosity solution of (1.4) with (1.3) is continuous and bounded in $\mathbf{R}^{n} \times[0, T)$.

We turn to the case where (1.2) is a level set equation. The following geometricity (H1), a notion originally introduced in [3], is a basic assumption on $H$ in the sense that $H$ derived from the associated surface evolution equation always satisfies it.

(H1) $H(x, \lambda p)=\lambda H(x, p)$ for all $(x, p) \in \mathbf{R}^{n} \times \mathbf{R}^{n}$ and $\lambda>0$.

Note that $(\mathrm{H} 1)$ implies that $H(x, 0)=0$ for all $x \in \mathbf{R}^{n}$. One of important properties of geometric equations is invariance under changes of dependent variables. 
Theorem 2.2 (Invariance [8, Theorem 4.2.1]). Assume (H1). Let $\theta: \mathbf{R} \rightarrow \mathbf{R}$ be a nondecreasing and upper semicontinuous (resp. lower semicontinuous) function. If $w$ is a viscosity subsolution (resp. supersolution) of (1.2), then so is $\theta \circ u$.

This invariance implies a uniqueness result of evolutions. Namely, if two initial data $u_{01}$ and $u_{02}$ share the same zero level, then so do the solutions $w_{1}$ and $w_{2}$. For the details see [8, Section 4.2.3, 4.2.4].

Another corollary of Theorem 2.2 is as follows. Let $\chi_{S}$ denote the characteristic function of a set $S$, i.e., $\chi_{s}(x)=1$ if $x \in S$ and $\chi_{s}(x)=0$ if $x \notin S$. We abbreviate $\{(x, t) \in$ $\left.\mathbf{R}^{n} \times(0, T) \mid w(x, t) \geqq 0\right\}$ to $\{w \geqq 0\}$ unless any confusion occurs.

Corollary 2.3. Assume (H1). If $w$ is a viscosity subsolution (resp. supersolution) of (1.2), then so is $\chi_{\{w \geqq 0\}}$ (resp. $\left.\chi_{\{w>0\}}\right)$.

Proof. Choosing an upper semicontinuous $\theta=\chi_{[0, \infty)}$, we have $\chi_{\{w \geqq 0\}}=\theta \circ w$. Similarly, $\chi_{\{w>0\}}=\theta \circ w$ for $\theta=\chi_{(0, \infty)}$. Thus Theorem 2.2 gives the results.

We prepare a sub- and supersolution of (1.4) of a simple form.

Lemma 2.4. Assume (H1). Let $c>0, M \geqq\|G\|$ and define $w^{ \pm}(x, t)=c e^{ \pm M t}$. Then $w^{+}$ (resp. $w^{-}$) is a classical supersolution (resp. subsolution) of (1.4), and $-w^{+}\left(\right.$resp. $-w^{-}$) is a classical subsolution (resp. supersolution) of (1.4).

Proof. Recalling that $H(x, 0)=0$ by $(\mathrm{H} 1)$, we compute

$$
\partial_{t} w^{+}+H\left(x, \nabla w^{+}\right)=M c e^{M t}+H(x, 0)=M w^{+}+0 \geqq w^{+} G\left(x, \nabla w^{+}\right),
$$

and thus $w^{+}$is a supersolution. The rest assertions follow in the same manner.

The next proposition guarantees that the zero level set is not distorted by our improved equation (1.4). This is, at least formally, expected to be true since (1.4) is the same equation as (1.2) at the zero level $\{u=0\}$.

Proposition 2.5 (Preservation of the zero level set). Assume (H1). Let $w$ and $u$ be, respectively, a viscosity solution of (1.2) and (1.4) with the same initial condition (1.3). Then we have $\{w=0\}=\{u=0\}$ and $\{ \pm w>0\}=\{ \pm u>0\}$.

Here we do not impose a special structure on $G$ in (1.4); we only need (CP). The proof can be found in [11, Theorem 3.1], where the result is shown for more general equations including (1.9). There, boundedness of $\beta$ is assumed, but this is not a restrictive condition when we study a bounded solution like the current case.

Since we have already known uniqueness of evolutions for (1.2), as a corollary of Proposition 2.5, we obtain uniqueness of evolutions for (1.4) at the zero level.

\section{Comparison with the signed distance function}

We study an evolution of the zero level set $\{\Gamma(t)\}_{t \in[0, T)}$ associated with (1.2) and (1.3). In the following we assume

(S1) $\Gamma(t) \subset \mathbf{R}^{n}$ is bounded uniformly in $t \in[0, T)$. Here $\Gamma(t)$ is given by (1.1), and $w$ is the unique viscosity solution to (1.2) with (1.3). 
This boundedness of $\Gamma(t)$ is not an essential assumption but slightly weakens an assumption on smoothness of $H$. For this, see the comment after (H2).

For the solution $w$ in (S1), we define the signed distance function $d: \mathbf{R}^{n} \times[0, T) \rightarrow \mathbf{R}$ as in (1.7). For later use we further set $\Gamma:=\left\{(x, t) \in \mathbf{R}^{n} \times(0, T) \mid w(x, t)=0\right\}$ and $D^{ \pm}:=\left\{(x, t) \in \mathbf{R}^{n} \times(0, T) \mid \pm w(x, t)>0\right\}$.

We intend to prove that, under a suitable choice of $G$, a viscosity solution $u$ of (1.4) satisfies the estimate (1.8) involving $d$. This shows that the slope of $u$ is close to one near the zero level. For this purpose, we first derive the equation for $d$.

In this paper we assume that $d$ is smooth near the zero level set, and so the derivatives of $d$ are interpreted in the classical sense. It is future work to extend the results below to a non-smooth case. Our assumption concerning smoothness is

(S2) There exist a constant $\delta \in(0,1)$ such that

(i) $d \in C^{1}(\mathcal{N})$, where $\mathcal{N}:=\left\{(x, t) \in \mathbf{R}^{n} \times(0, T)|| d(x, t) \mid<\delta\right\}$;

(ii) For every $(x, t) \in \mathcal{N}$, we have $\bar{x}:=x-d(x, t) \nabla d(x, t) \in \Gamma(t), \partial_{t} d(x, t)=\partial_{t} d(\bar{x}, t)$ and $\nabla d(x, t)=\nabla d(\bar{x}, t)$.

Here $\bar{x}$ represents the perpendicular foot on $\Gamma(t)$ from $x$. It is known that (S2) is true if $\Gamma$ is smooth enough, e.g., if $\Gamma$ is in $C^{2,1}$-class $\left(C^{2}\right.$ in $x$ and $C^{1}$ in $\left.t\right)$. See [12, Theorem 3.8, Theorem 5.14] for instance. Instead of assuming sufficient smoothness of interfaces, we assume (S2) which is really needed in discussions below.

Lemma 3.1. Assume (H1) and (S2). Then, for all $(x, t) \in \mathcal{N}$,

$$
\partial_{t} d(x, t)+H(x-d(x, t) \nabla d(x, t), \nabla d(x, t))=0 .
$$

Proof. We need to prove $\partial_{t} d(\bar{x}, t)+H(\bar{x}, \nabla d(\bar{x}, t))=0$ for $\bar{x}=x-d(x, t) \nabla d(x, t)$. Consider the characteristic function $\chi_{\{w>0\}}$, where $w$ is the solution in (S1). By Corollary 2.3 this $\chi_{\{w>0\}}$ is a supersolution of (1.2). Now, since $\bar{x} \in \Gamma(t)$, we have $\chi_{\{w>0\}}(\bar{x}, t)=d(\bar{x}, t)=0$. Moreover, $d \leqq \chi_{\{w>0\}}$ in $\mathcal{N}$. These facts show that $d \in C^{1}(\mathcal{N})$ is a test function for $\chi_{\{w>0\}}$ at $(\bar{x}, t)$. By the definition of viscosity supersolutions, we obtain $\partial_{t} d(\bar{x}, t)+H(\bar{x}, \nabla d(\bar{x}, t)) \geqq$ 0 . The opposite inequality is derived in a similar way by considering $\chi_{\{w \geqq 0\}}-1$.

Remark 1. Even if $d$ is not smooth, it is known that $d$ is a viscosity supersolution of (3.1) in $D^{+}$. Also, if $\phi$ is a test function for $d$ at $(x, t) \in D^{+}$from below, then $|\nabla \phi(x, t)|=1$ See, e.g., [5, Proof of Theorem 2.2, Step 1-3], [1, Proposition II.2.14] for them. In particular, if $d$ is differentiable at $(x, t)$, then $|\nabla d(x, t)|=1$.

Let us rewrite $(3.1)$ as follows. We first insert $H(x, \nabla d)$ and then apply Taylor (linear) approximation to obtain

$$
\begin{aligned}
\partial_{t} d+H(x, \nabla d) & =H(x, \nabla d)-H(x-d \nabla d, \nabla d) \\
& =d\left\langle\nabla_{x} H(x, \nabla d), \nabla d\right\rangle+o(d) .
\end{aligned}
$$

If we drop the error term $o(d)$, then (3.3) is of the form (1.4). For this reason it is reasonable to define $G(x, p)$ in (1.4) on the basis of $\left\langle\nabla_{x} H(x, p), p\right\rangle$.

We shall give precise assumptions on $H$ and $G$. Set $S^{n-1}:=\left\{x \in \mathbf{R}^{n}|| x \mid=1\right\}$.

(H2) For any compact set $K \subset \mathbf{R}^{n}$ there is a modulus $\omega_{K}$ such that

$$
\left|H(x, p)-H(x-h p, p)-h\left\langle\nabla_{x} H(x, p), p\right\rangle\right| \leqq|h| \omega_{K}(|h|)
$$

for all $(x, p) \in K \times S^{n-1}$ and $h \in(0,1)$. 
This is true if $H(\cdot, p) \in C^{1}\left(\mathbf{R}^{n}\right)$ for all $p \in S^{n-1}$. For later use, we remark that

- We may assume that $\omega_{K}$ in (H2) is increasing and $\lim _{r \rightarrow \infty} \omega_{K}(r)=\infty$. Denote the inverse function of $\omega_{K}$ by $a=\omega_{K}^{-1}$, which is a modulus too.

We use the function $a$ in discussions below.

If a unbounded evolution is considered, one has to assume (H2) for $K=\mathbf{R}^{n}$, or assume $H(\cdot, p) \in C^{1}\left(\mathbf{R}^{n}\right)$ with $\nabla_{x} H(\cdot, p) \in U C\left(\mathbf{R}^{n}\right)$ for all $p \in S^{n-1}$.

We next state how to define $G: \mathbf{R}^{n} \times \mathbf{R}^{n} \rightarrow \mathbf{R}$ in the improved equation (1.4). The equation (3.3) tempts us to set $G(x, p)$ as $\left\langle\nabla_{x} H(x, p), p\right\rangle$; however, such a $G$ does not necessarily possess boundedness required in (CP). For this reason, we define $G$ as a suitable modification of the inner product; we replace $p$ by $p /|p|$.

(G1) There exists some $\sigma \in(0,1)$ such that

$$
G(x, p)=\left\langle\nabla_{x} H\left(x, \frac{p}{|p|}\right), \frac{p}{|p|}\right\rangle \quad \text { for all }(x, p) \in \mathbf{R}^{n} \times \mathbf{R}^{n},|p| \geqq \sigma .
$$

The constant $\sigma>0$ is introduced to avoid a singularity at $p=0$. Any conditions for $|p|<\sigma$ are not required in (G1). However, recall that we are now assuming that $G$ satisfies the continuity given in (CP) in the whole of $\mathbf{R}^{n} \times \mathbf{R}^{n}$.

For simplification we collect all the assumptions above.

(A1) $H$ and $G$ satisfy (CP), (H1), (H2) and (G1); moreover, (S1) and (S2) hold.

By the effect of the error term $o(d)$, the distance function $d$ itself is neither a subsolution nor a supersolution of (1.4). To get such solutions, we set

$$
d^{+}(x, t):=e^{\varepsilon t} d(x, t), \quad d^{-}(x, t):=e^{-\varepsilon t} d(x, t) .
$$

Proposition 3.2 (Sub- and supersolutions near zero level sets). Assume (A1). Choose $\varepsilon, K$, a so that

$$
\varepsilon \in\left(0,-\frac{\log \sigma}{T}\right] ; K \subset \mathbf{R}^{n} \text { is a compact set such that } \mathcal{N} \subset K ; a=\omega_{K}^{-1},
$$

where $\sigma$ is the constant in (G1) and $\omega_{K}$ is the modulus in (H2). Let $r>0$ and set $\delta_{r}:=$ $\min \{a(r), \delta\}$. Then, for all $(x, t) \in\left\{|d|<\delta_{r}\right\}$,

$$
\begin{aligned}
& \left|\partial_{t} d^{+}(x, t)+H\left(x, \nabla d^{+}(x, t)\right)-d^{+}(x, t)\left\{G\left(x, \nabla d^{+}(x, t)\right)+\varepsilon\right\}\right| \leqq r\left|d^{+}(x, t)\right|, \\
& \left|\partial_{t} d^{-}(x, t)+H\left(x, \nabla d^{-}(x, t)\right)-d^{-}(x, t)\left\{G\left(x, \nabla d^{-}(x, t)\right)-\varepsilon\right\}\right| \leqq r\left|d^{-}(x, t)\right| .
\end{aligned}
$$

In particular, if $r \leqq \varepsilon$, then $d^{+}$is a supersolution (resp. subsolution) of (1.4) in $\{0 \leqq d<$ $\left.\delta_{r}\right\}$ (resp. in $\left\{-\delta_{r}<d \leqq 0\right\}$ ), and $d^{-}$is a subsolution (resp. supersolution) of (1.4) in $\left\{0 \leqq d<\delta_{r}\right\}$ (resp. in $\left.\left\{-\delta_{r}<d \leqq 0\right\}\right)$.

Proof. Let us fix $(x, t) \in\left\{|d|<\delta_{r}\right\}$. Then $d$ is differentiable at $(x, t)$ since $\delta_{r} \leqq \delta$. Using (H1) and (3.2), we compute

$$
\begin{aligned}
\partial_{t} d^{ \pm}+H\left(x, \nabla d^{ \pm}\right) & = \pm \varepsilon d^{ \pm}+e^{ \pm \varepsilon t} \partial_{t} d+H\left(x, e^{ \pm \varepsilon t} \nabla d\right) \\
& = \pm \varepsilon d^{ \pm}+e^{ \pm \varepsilon t}\left\{\partial_{t} d+H(x, \nabla d)\right\} \\
& = \pm \varepsilon d^{ \pm}+e^{ \pm \varepsilon t}\{H(x, \nabla d)-H(x-d \nabla d, \nabla d)\}
\end{aligned}
$$


Now, the choice of $\varepsilon$ gives $e^{-\varepsilon t}>e^{-\varepsilon T} \geqq \sigma$ for all $t \in(0, T)$. Thus (G1) implies

$$
G\left(x, \nabla d^{ \pm}\right)=G\left(x, e^{ \pm \varepsilon t} \nabla d\right)=\left\langle\nabla_{x} H(x, \nabla d), \nabla d\right\rangle .
$$

Here we used $|\nabla d|=1$ (Remark 1). We next choose $h=d(x, t)$ and $p=\nabla d(x, t)$ in (3.4). Since $|h|<\delta<1$ and $|p|=1$, these choices are possible. Then

$$
\begin{aligned}
\left|H(x, \nabla d)-H(x-d \nabla d, \nabla d)-d\left\langle\nabla_{x} H(x, \nabla d), \nabla d\right\rangle\right| & \leqq|d| \omega_{K}(|d|) \leqq|d| \omega_{K}(a(r)) \\
& =r|d| .
\end{aligned}
$$

We apply (3.8) to the left-hand side and multiply both the sides by $e^{ \pm \varepsilon t}$ to get

$$
\left|e^{ \pm \varepsilon t}\{H(x, p)-H(x-d \nabla d, \nabla d)\}-d^{ \pm} G\left(x, \nabla d^{ \pm}\right)\right| \leqq r\left|d^{ \pm}\right| .
$$

Finally, combining (3.9) and (3.7), we arrive at both the equations that we have to prove. The remaining assertions for $r \leqq \varepsilon$ are immediate consequences.

In order to derive the estimates (1.8) from the comparison principle, we need to extend the local sub-/supersolution $d^{ \pm}$in Proposition 3.2 so that they become a sub-/supersolution in the whole of $\mathbf{R}^{n} \times(0, T)$. To do this, we prepare

Lemma 3.3. Let $f_{1}, f_{2}, \phi \in C^{1}(U)$ for $U \subset \mathbf{R}^{n}$ open, and define $g:=\min \left\{f_{1}, f_{2}\right\}$. Assume that $f_{1}(z)=f_{2}(z)$ at $z \in U$. If $g-\phi$ attains a local maximum at $z$, then $\nabla \phi(z)=$ $\lambda \nabla f_{1}(z)+(1-\lambda) \nabla f_{2}(z)$ for some $\lambda \in[0,1]$.

Proof. By [1, Proposition II.2.13 and II.4.4], $D^{+} g(z)$, a superdifferential of $g$ at $z$, is equal to the convex hull of $Y(z):=\left\{\nabla f_{1}(z), \nabla f_{2}(z)\right\}$. This gives the lemma.

Let $c, L, M>0$. We set $V(x, t):=(3 L / c) e^{\|G\| t} d^{+}(x, t)$ and define $u^{ \pm}: \mathbf{R}^{n} \times[0, T) \rightarrow \mathbf{R}$, which is shown in Figure 1, as

$$
\begin{aligned}
& u^{+}(x, t)= \begin{cases}\min \left\{\max \left\{d^{+}(x, t), V(x, t)-L\right\}, L e^{\|G\| t}\right\} & \text { if } d(x, t) \geqq 0, \\
\max \left\{d^{-}(x, t),-c e^{-M t}\right\} & \text { if } d(x, t)<0,\end{cases} \\
& u^{-}(x, t)= \begin{cases}\min \left\{d^{-}(x, t), c e^{-M t}\right\} & \text { if } d(x, t) \geqq 0, \\
\max \left\{\min \left\{d^{+}(x, t), V(x, t)+L\right\},-L e^{\|G\| t}\right\} & \text { if } d(x, t)<0 .\end{cases}
\end{aligned}
$$

Proposition 3.4 (Extension of sub- and supersolutions). Assume (A1). Choose $\varepsilon, K, a$ as in (3.6). Let $c, L, M>0$ be constants such that $0<c \leqq \min \{a(\varepsilon), \delta\}, L \geqq c, M \geqq$ $2\|G\| /\left(1-\sigma e^{\varepsilon T}\right)$ and $M>\varepsilon$. Define $d^{ \pm}$as in (3.5). Then, $u^{+}$and $u^{-}$above are, respectively, a viscosity supersolution and a viscosity subsolution of (1.4) in $\mathbf{R}^{n} \times(0, T)$.

Proof. 1. We only prove that $u^{+}$and $u^{-}$are, respectively, a supersolution and a subsolution in $\{d \geqq 0\}$; the proof in $\{d<0\}$ is the same as that in $\{d>0\}$.

On $\{d=0\}=\Gamma$ the viscosity properties are derived in the same manner as in the proof of Lemma 3.1. In fact, a test function for $u^{+}$at $(z, s) \in \Gamma$ is also a test function for $\chi_{\{w>0\}}$. Therefore $\partial_{t} \phi(z, s)+H(z, \nabla \phi(z, s)) \geqq 0=u^{+}(z, s) G(z, \nabla \phi(z, s))$. The proof for $u^{-}$is similar. 


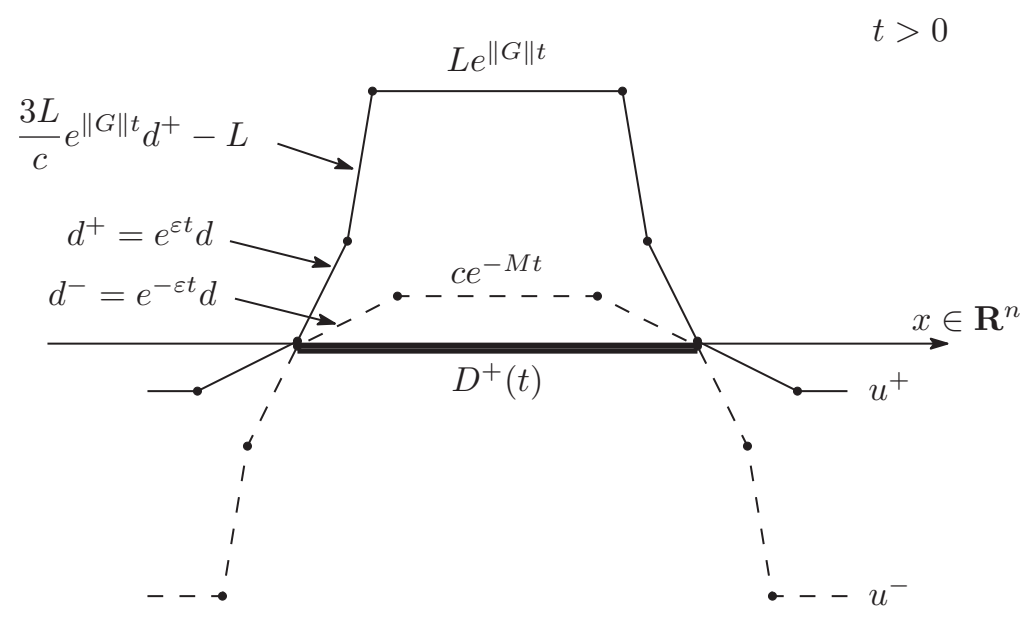

Figure 1: Definitions of $u^{+}$and $u^{-}$.

In $\{d \geqq 0\}$, using $L \geqq c$, we see that $u^{+}$and $u^{-}$are represented as follows:

$$
\begin{aligned}
& u^{+}(x, t)= \begin{cases}d^{+}(x, t) & \text { if } 0 \leqq d^{+}(x, t) \leqq c L /\left(3 L e^{\|G\| t}-c\right), \\
V(x, t)-L & \text { if } c L /\left(3 L e^{\|G\| t}-c\right) \leqq d^{+}(x, t) \leqq c\left(1+e^{-\|G\| t}\right) / 3, \\
L e^{\|G\| t} & \text { if } c\left(1+e^{-\|G\| t}\right) / 3 \leqq d^{+}(x, t),\end{cases} \\
& u^{-}(x, t)= \begin{cases}d^{-}(x, t) & \text { if } 0 \leqq d^{-}(x, t) \leqq c e^{-M t}, \\
c e^{-M t} & \text { if } c e^{-M t} \leqq d^{-}(x, t) .\end{cases}
\end{aligned}
$$

When $0 \leqq d^{+}(x, t) \leqq c\left(1+e^{-\|G\| t}\right) / 3$, we have

$$
0 \leqq d(x, t) \leqq \frac{c\left(1+e^{-\|G\| t}\right) e^{-\varepsilon t}}{3}<\frac{2}{3} c \leqq \frac{2}{3} \min \{a(\varepsilon), \delta\} .
$$

It thus follows from Proposition 3.2 with $r=\varepsilon$ that $d^{+}$is a classical supersolution of (1.4) in $\left\{0 \leqq d^{+} \leqq c\left(1+e^{-\|G\| t}\right) / 3\right\}$. Similarly, if $0 \leqq d^{-}(x, t) \leqq c e^{-M t}$, then

$$
0 \leqq d(x, t) \leqq c e^{(\varepsilon-M) t}<c \leqq \min \{a(\varepsilon), \delta\}
$$

since $M>\varepsilon$. Hence $d^{-}$is a classical subsolution of (1.4) in $\left\{0 \leqq d^{-} \leqq c e^{-M t}\right\}$.

2. $u^{-}$is a viscosity subsolution of (1.4) in $\{d>0\}$. Since we have $M \geqq 2\|G\| /(1-$ $\left.\sigma e^{\varepsilon T}\right)>2\|G\| \geqq\|G\|$, Lemma 2.4 implies that $u(x, t)=c e^{-M t}$ is a subsolution in $\left\{c e^{-M t}<\right.$ $\left.d^{-}\right\}$. What is left to show is that $u^{-}$is a subsolution on $\left\{d^{-}=c e^{-M t}\right\}$. To do this, take a test function $\phi$ for $u^{-}$at $(z, s) \in\left\{d^{-}=c e^{-M t}\right\}$. Set $(p, \tau):=\left(\nabla \phi(z, s), \partial_{t} \phi(z, s)\right)$ and $\alpha:=d^{-}(z, s)=c e^{-M s}$. We want to deduce that $I:=\tau+H(z, p)-\alpha G(z, p) \leqq 0$. Applying Lemma 3.3 to $u^{-}$, we see

$$
\begin{aligned}
& p=\lambda \nabla d^{-}(z, s)+(1-\lambda) \nabla\left(c e^{-M t}\right)(z, s)=\lambda \nabla d^{-}(z, s), \\
& \tau=\lambda \partial_{t} d^{-}(z, s)+(1-\lambda) \partial_{t}\left(c e^{-M t}\right)(z, s)=\lambda \partial_{t} d^{-}(z, s)-M(1-\lambda) \alpha
\end{aligned}
$$


for some $\lambda \in[0,1]$, and thus

$$
\begin{aligned}
I & =\lambda \partial_{t} d^{-}-M(1-\lambda) \alpha+H\left(z, \lambda \nabla d^{-}\right)-\alpha G\left(z, \lambda \nabla d^{-}\right) \\
& =\lambda\left\{\partial_{t} d^{-}+H\left(z, \nabla d^{-}\right)\right\}-\alpha G\left(z, \lambda \nabla d^{-}\right)-M(1-\lambda) \alpha \\
& \leqq \lambda \alpha G\left(z, \nabla d^{-}\right)-\alpha G\left(z, \lambda \nabla d^{-}\right)-M(1-\lambda) \alpha .
\end{aligned}
$$

We now divide the situation into two different cases.

Case 1: $\left|\lambda \nabla d^{-}(z, s)\right| \geqq \sigma$. Then $G\left(z, \lambda \nabla d^{-}(z, s)\right)=G\left(z, \nabla d^{-}(z, s)\right)$ by (G1); thus

$$
I / \alpha=\lambda G\left(z, \nabla d^{-}\right)-G\left(z, \nabla d^{-}\right)-M(1-\lambda)=(1-\lambda)\left\{-G\left(z, \nabla d^{-}\right)-M\right\} .
$$

Recalling $M \geqq\|G\|$, we see that $I \leqq 0$.

Case 2: $\left|\lambda \nabla d^{-}(z, s)\right|<\sigma$. We first remark that $\lambda<\sigma /\left|\nabla d^{-}(z, s)\right|=\sigma e^{\varepsilon s}<\sigma e^{\varepsilon T}$. Using this estimate, we observe

$$
I / \alpha \leqq \lambda\|G\|+\|G\|-M\left(1-\sigma e^{\varepsilon T}\right) \leqq 2\|G\|-M\left(1-\sigma e^{\varepsilon T}\right) .
$$

The right-hand side is nonpositive by the choice of $M$, and therefore $I \leqq 0$.

3. $u^{+}$is a supersolution of (1.4) in $\{d>0\}$. We first claim that, if $K \geqq 1$ and $\eta>0$, then $V_{0}(x, t):=K e^{\|G\| t} d^{+}(x, t)-\eta$ is a classical supersolution of (1.4) in $\left\{V_{0} \geqq 0\right\} \cap\{d<c\}$. Fix $(x, t)$ such that $V_{0}(x, t) \geqq 0$ and $d(x, t)<c$. Then $\left|\nabla V_{0}\right|=K e^{\|G\| t}\left|\nabla d^{+}\right| \geqq\left|\nabla d^{+}\right| \geqq 1$, and so $G\left(x, \nabla V_{0}\right)=G\left(x, \nabla d^{+}\right)$by (G1). Since $d^{+}$is a supersolution of (1.4), the following computation shows the claim:

$$
\begin{aligned}
\partial_{t} V_{0}+H\left(x, \nabla V_{0}\right) & =K\left\{\|G\| e^{\|G\| t} d^{+}+e^{\|G\| t} \partial_{t} d^{+}\right\}+H\left(x, K e^{\|G\| t} \nabla d^{+}\right) \\
& =\|G\|\left(V_{0}+\eta\right)+K e^{\|G\| t}\left\{\partial_{t} d^{+}+H\left(x, \nabla d^{+}\right)\right\} \\
& \geqq\|G\|\left(V_{0}+\eta\right)+K e^{\|G\| t} d^{+} G\left(x, \nabla d^{+}\right) \\
& =\left(V_{0}+\eta\right)\left\{\|G\|+G\left(x, \nabla V_{0}\right)\right\} \\
& \geqq V_{0}\left\{\|G\|+G\left(x, \nabla V_{0}\right)\right\} \geqq V_{0} G\left(x, \nabla V_{0}\right) .
\end{aligned}
$$

Hereafter we choose $K=3 L / c$ and $\eta=L$, so that $V_{0}(x, t)=V(x, t)-L$.

Since we have already shown that $d^{+}, V_{0}$ and $L e^{\|G\| t}$ are supersolutions, we only need to study $u^{+}$on $\left\{d^{+}=V_{0}\right\}$ and $\left\{V_{0}=L e^{\|G\| t}\right\}$. On $\left\{V_{0}=L e^{\|G\| t}\right\}$ it is easily seen that there is no test function for $u^{+}$from below, and so $u^{+}$is a supersolution.

We next assume that $\phi$ is a test function for $u^{+}$at $(z, s) \in\left\{d^{+}=V_{0}\right\}$. Set $(p, \tau):=$ $\left(\nabla \phi(z, s), \partial_{t} \phi(z, s)\right)$ and $\beta:=d^{+}(z, s)=V_{0}(z, s)$. Our goal is to show that $J:=\tau+H(z, p)-$ $\beta G(z, p) \geqq 0$. Similarly to Step 2 , there is $\lambda \in[0,1]$ such that

$$
\begin{aligned}
p & =\lambda \nabla d^{+}(z, s)+(1-\lambda) \nabla V_{0}(z, s)=\lambda \nabla d^{+}(z, s)+(1-\lambda) K e^{\|G\| s} \nabla d^{+}(z, s), \\
\tau & =\lambda \partial_{t} d^{+}(z, s)+(1-\lambda) \partial_{t} V_{0}(z, s) \\
& =\lambda \partial_{t} d^{+}(z, s)+(1-\lambda)\left\{\|G\|(\beta+\eta)+K e^{\|G\| s} \partial_{t} d^{+}(z, s)\right\}
\end{aligned}
$$

by Lemma 3.3. Set $\lambda^{\prime}:=\lambda+(1-\lambda) K e^{\|G\| s}$. Then

$$
p=\lambda^{\prime} \nabla d^{+}(z, s), \quad \tau=\lambda^{\prime} \partial_{t} d^{+}(z, s)+(1-\lambda)\|G\|(\beta+\eta) .
$$


Also, $\lambda^{\prime} \geqq \lambda+(1-\lambda)=1$, which gives $G\left(z, \lambda^{\prime} \nabla d^{+}\right)=G\left(z, \nabla d^{+}\right)$. Thus

$$
\begin{aligned}
J & =\lambda^{\prime} \partial_{t} d^{+}+(1-\lambda)\|G\|(\beta+\eta)+H\left(z, \lambda^{\prime} \nabla d^{+}\right)-\beta G\left(z, \lambda^{\prime} \nabla d^{+}\right) \\
& =\lambda^{\prime}\left\{\partial_{t} d^{+}+H\left(z, \nabla d^{+}\right)\right\}+(1-\lambda)\|G\|(\beta+\eta)-\beta G\left(z, \nabla d^{+}\right) \\
& \geqq \lambda^{\prime} \beta G\left(z, \nabla d^{+}\right)+(1-\lambda)\|G\|(\beta+\eta)-\beta G\left(z, \nabla d^{+}\right) \\
& =-\left(1-\lambda^{\prime}\right) \beta G\left(z, \nabla d^{+}\right)+(1-\lambda)\|G\|(\beta+\eta) .
\end{aligned}
$$

The definitions of $\lambda^{\prime}$ and $\beta$ yield $-\left(1-\lambda^{\prime}\right) \beta=(1-\lambda)\left(K e^{\|G\| s} \beta-\beta\right)=(1-\lambda) \eta$, and so

$$
\begin{aligned}
J & \geqq(1-\lambda) \eta G\left(z, \nabla d^{+}\right)+(1-\lambda)\|G\|(\beta+\eta) \\
& =(1-\lambda)\left[\eta\left\{G\left(z, \nabla d^{+}\right)+\|G\|\right\}+\beta\|G\|\right] \geqq 0 .
\end{aligned}
$$

This completes the proof.

Remark 2. Define

$$
\rho_{0}:=\min \left\{\frac{c L e^{-\varepsilon T}}{3 L e^{\|G\| T}-c}, c e^{(\varepsilon-M) T}\right\} .
$$

Then, by the definitions of $u^{+}$and $u^{-}$we see that

$$
\left(u^{+}(x, t), u^{-}(x, t)\right)= \begin{cases}\left(e^{\varepsilon t} d(x, t), e^{-\varepsilon t} d(x, t)\right) & \text { if } 0 \leqq d(x, t) \leqq \rho_{0}, \\ \left(e^{-\varepsilon t} d(x, t), e^{\varepsilon t} d(x, t)\right) & \text { if }-\rho_{0} \leqq d(x, t) \leqq 0 .\end{cases}
$$

We are now in a position to state our main theorem. There we take an initial data $u_{0}$ which agrees with the signed distance function near $\Gamma(0)$. Namely, we assume

$$
\text { there exists some } m>0 \text { such that } \begin{cases}u_{0}(x)=d_{0}(x) & \text { if }\left|d_{0}(x)\right| \leqq m, \\ u_{0}(x) \geqq m & \text { if } d_{0}(x)>m, \\ u_{0}(x) \leqq m & \text { if } d_{0}(x)<-m,\end{cases}
$$

where we set $d_{0}(x):=d(x, 0)$.

Theorem 3.5 (Comparison with the signed distance function). Assume (A1) and (3.14). Let $u$ be a viscosity solution of (1.4) with (1.3). Then, for every $\varepsilon>0$, there exists a positive constant $\rho(\varepsilon)>0$ such that

$$
\begin{array}{ll}
e^{-\varepsilon t} d(x, t) \leqq u(x, t) \leqq e^{\varepsilon t} d(x, t) & \text { if } 0 \leqq d(x, t) \leqq \rho(\varepsilon), \\
e^{\varepsilon t} d(x, t) \leqq u(x, t) \leqq e^{-\varepsilon t} d(x, t) & \text { if }-\rho(\varepsilon) \leqq d(x, t) \leqq 0 .
\end{array}
$$

Proof. We may take an $\varepsilon>0$ small enough. Choose $\varepsilon, K, a$ as in (3.6). Define

$$
c:=\min \{\delta, a(\varepsilon), m\}, L:=\left\|u_{0}\right\|=\sup _{x \in \mathbf{R}^{n}}\left|u_{0}(x)\right|, M:=\max \left\{\frac{2\|G\|}{1-\sigma e^{\varepsilon T}}, \frac{3 \varepsilon}{2}\right\},
$$

and let $u^{ \pm}: \mathbf{R}^{n} \times[0, T) \rightarrow \mathbf{R}$ be the functions in (3.10) and (3.11) with these constants. We shall prove

$$
u^{-}(x, 0) \leqq u_{0}(x) \leqq u^{+}(x, 0) \quad \text { for all } x \in \mathbf{R}^{n}
$$


We give a discussion only in $\left\{d_{0}(x) \geqq 0\right\}$; similar arguments work in $\left\{d_{0}(x)<0\right\}$. Let $x \in \mathbf{R}^{n}$ satisfy $d_{0}(x) \geqq 0$. By the definitions of $u^{ \pm}$, we notice that

$$
\begin{aligned}
& u^{+}(x, 0)=\min \left\{\max \left\{d_{0}(x), \frac{3\left\|u_{0}\right\|}{c} d_{0}(x)-\left\|u_{0}\right\|\right\},\left\|u_{0}\right\|\right\}, \\
& u^{-}(x, 0)=\min \left\{d_{0}(x), c\right\} .
\end{aligned}
$$

If $0 \leqq d_{0}(x) \leqq m$, then $u_{0}(x)=d_{0}(x)$ by $(3.14)$ and hence

$$
\begin{aligned}
& u^{+}(x, 0) \geqq \min \left\{d_{0}(x),\left\|u_{0}\right\|\right\}=\min \left\{u_{0}(x),\left\|u_{0}\right\|\right\}=u_{0}(x), \\
& u^{-}(x, 0) \leqq \min \left\{d_{0}(x), m\right\}=d_{0}(x)=u_{0}(x) .
\end{aligned}
$$

In the case where $d_{0}(x)>m$, we estimate

$$
\begin{aligned}
& u^{+}(x, 0) \geqq \min \left\{\frac{3\left\|u_{0}\right\|}{c} d_{0}(x)-\left\|u_{0}\right\|,\left\|u_{0}\right\|\right\} \geqq \min \left\{2\left\|u_{0}\right\|,\left\|u_{0}\right\|\right\} \geqq u_{0}(x), \\
& u^{-}(x, 0) \leqq \min \left\{d_{0}(x), m\right\}=m \leqq u_{0}(x) .
\end{aligned}
$$

Thus (3.15) is proved. The comparison principle (Theorem 2.1) for (3.15) implies

$$
u^{-}(x, t) \leqq u(x, t) \leqq u^{+}(x, t) \text { for all }(x, t) \in \mathbf{R}^{n} \times(0, T) .
$$

We set $\rho(\varepsilon):=\rho_{0}$, where $\rho_{0}$ is the constant in (3.12) with $c, L$ and $M$ given as above. (Actually, $\rho(\varepsilon)$ depends on $T,\|G\|, \sigma, a, m$, and $\left\|u_{0}\right\|$ too.) Finally, combing (3.13) and (3.16) gives the conclusion of the theorem.

\section{Examples and numerical results}

After giving examples of $G=G(x, p)$ appearing in (1.4), we show numerical results.

Example 1. We consider the transport equation (1.5) with $H(x, p)=\langle X(x), p\rangle$. The gradient of $H$ with respect to $x$ is $\nabla_{x} H(x, p)=X^{\prime}(x) p$, and so

$$
G(x, p)=\left\langle\nabla_{x} H\left(x, \frac{p}{|p|}\right), \frac{p}{|p|}\right\rangle=\frac{\left\langle X^{\prime}(x) p, p\right\rangle}{|p|^{2}} \quad \text { if }|p| \geqq \sigma .
$$

This is nothing but the function on the right-hand side of (1.6) by Roisman.

Example 2. Let $H(x, p)=c(x)|p|$. Then (1.2) is the eikonal equation:

$$
\partial_{t} w(x, t)+c(x)|\nabla w(x, t)|=0 .
$$

Since the gradient of $H$ is $\nabla_{x} H(x, p)=|p| \nabla c(x)$, we see that

$$
G(x, p)=\left\langle\nabla_{x} H\left(x, \frac{p}{|p|}\right), \frac{p}{|p|}\right\rangle=\frac{\langle\nabla c(x), p\rangle}{|p|} \quad \text { if }|p| \geqq \sigma .
$$

Example 3. We show a numerical comparison between solutions to the original (1.2) and the improved (1.4) in the two dimensional case $n=2$. The following computations are by courtesy of Professor Norikazu Yamaguchi. 
We consider the eikonal equation (4.1) with $c\left(x_{1}, x_{2}\right)=-f\left(x_{2}\right)=-\tanh x_{2}$ under the initial data $u_{0}\left(x_{1}, x_{2}\right)=-x_{1}$; namely, the initial zero level is $x_{2}$-axis. In this case, the slope of the solution $w$ of (1.2) goes to $\infty$ at the zero level as is shown in Figure 2, where five black curves represent the levels of $w$ at $-0.2,-0.1,0,0.1,0.2$. On the other hand, for the solution $u$ of (1.4), we can see that the level sets have been well separated for a while as in Figure 3.

The computations are done by the characteristic Galerkin method ([14]), in which we rewrite the equations (1.2) and (1.4) as nonlinear transport equations:

$$
\left\{\begin{array}{l}
\partial_{t} w+\langle\mathbf{v}(w), \nabla w\rangle=0, \\
\partial_{t} u+\langle\mathbf{v}(u)+\mathbf{w}(u), \nabla u\rangle=0
\end{array} \quad \text { with } \mathbf{v}(u)=-f\left(x_{2}\right) \frac{\nabla u}{|\nabla u|}, \mathbf{w}(u)=u \frac{f^{\prime}\left(x_{2}\right)}{|\nabla u|} \mathbf{e}_{2},\right.
$$

respectively. Here $\mathbf{e}_{2}=(0,1) \in \mathbf{R}^{2}$.

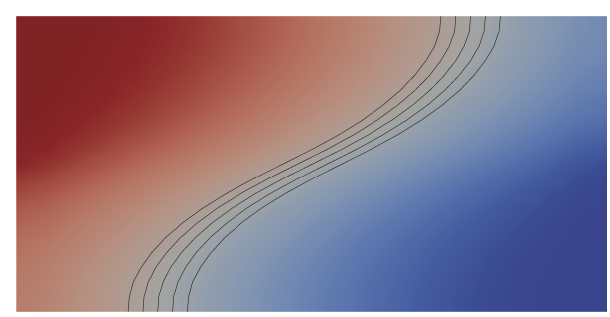

(a) $t=1.5$

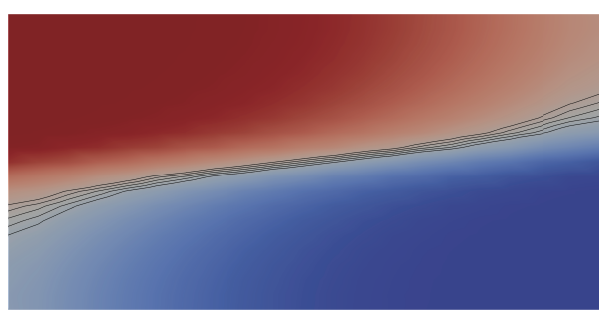

(b) $t=3.5$

Figure 2: Level sets of the solution $w$ of the original equation $(1.2)$ in $[-2,2] \times[-1,1]$.

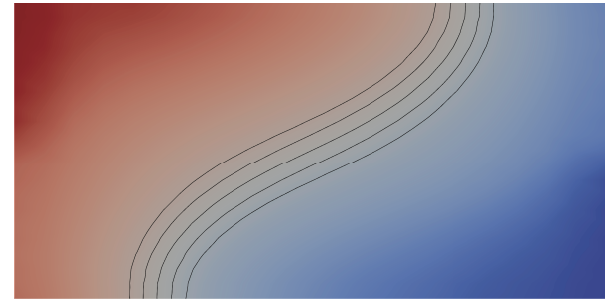

(a) $t=1.5$

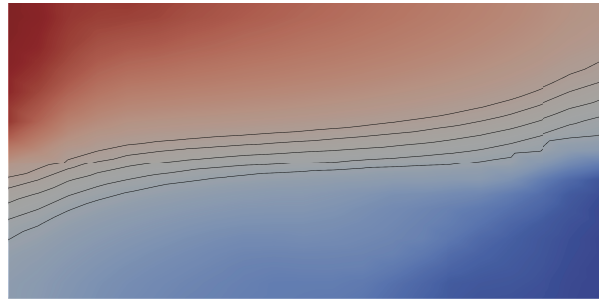

(b) $t=3.5$

Figure 3: Level sets of the solution $u$ of the improved equation $(1.4)$ in $[-2,2] \times[-1,1]$.

\section{$5 \quad$ Rate of convergence for (1.9)}

For the solution $u^{\theta}$ of (1.9), we shall derive a bound by the distance function $d$. The conditions assumed in [11, Theorem 2.2] to show (1.10) are

(A2) $u_{0} \in \operatorname{Lip}\left(\mathbf{R}^{n}\right)$ (Lipschitz continuous); $H$ satisfies (CP) (i) with $\omega(r)=L_{1} r\left(L_{1}>0\right)$ and (H1); $\beta \in \operatorname{Lip}(\mathbf{R})$ is non-decreasing and bounded in $\mathbf{R}$ with $\pm \beta(r)>0$ if $\pm r>0$; $h \in U C\left(\mathbf{R}^{n}\right)$ and $h(p)>0$ if $|p|<1, h(p)<0$ if $|p|>1$. 
We emphasize that in [11] any regularities of $d$ like (S2) are not needed for (1.10); $d$ can be discontinuous in $t$. Note also that the current assumptions on $H$ guarantee

$$
|H(x, p)-H(x-h p, p)| \leqq L_{1} \min \{3 h, 2\} \quad \text { for all } x \in \mathbf{R}^{n}, p \in S^{n-1} \text { and } h>0 .
$$

In fact, (2.1) gives the bound $3 h L_{1}$ while $2 L_{1}$ is derived since $|H| \leqq L_{1}$ in $\mathbf{R}^{n} \times S^{1}$. This comes from $H(x, 0)=0$ and (2.1). Concerning $\beta$, we further assume

(B1) There exist some $b_{1}, \mu_{1}>0$ such that $b_{1}|r| \leqq|\beta(r)|$ for all $r \in\left[-\mu_{1}, \mu_{1}\right]$.

We now define functions $g:[0,1] \rightarrow[0, g(1)]$ and $\tilde{g}:[0, g(1)] \rightarrow[0,1]$ as

$$
g(r):=\min \{-h(p)|1+r \leqq| p \mid \leqq 2\}, \quad \tilde{g}(s):=\inf \{r \in[0,1] \mid g(r) \geqq s\} .
$$

Then $g(0)=0, g>0$ in $(0,1], g$ is continuous and non-decreasing in $[0,1]$. By these properties we have $\tilde{g}>0$ in $(0, g(1)], g \circ \tilde{g}=\mathrm{id}$ and $\lim _{s \rightarrow 0} \tilde{g}(s)=\tilde{g}(0)=0$.

Theorem 5.1. Assume (A2) and (B1). Let $\alpha \in(0,1)$. Set $\rho(\theta):=\tilde{g}\left(\theta^{-\alpha}\right)$ and $v^{\theta}(x, t):=$ $(1+\rho(\theta)) d(x, t)$. Then $v^{\theta}$ is a viscosity supersolution (resp. subsolution) of (1.9) in $D^{+}$ (resp. in $D^{-}$) for all $\theta>0$ sufficiently large. Moreover, if $\left|u_{0}\right| \leqq\left|d_{0}\right|$ in $\mathbf{R}^{n}$, then the unique solution $u^{\theta}$ of (1.9) with (1.3) satisfies $\left|u^{\theta}\right| \leqq\left|v^{\theta}\right|$ in $\mathbf{R}^{n} \times(0, T)$ for such $\theta>0$.

Proof. We prove that $v^{\theta}$ is a supersolution in $D^{+}$; the discussion is similar in $D^{-}$. Take a test function $\phi$ for $v^{\theta}$ at $(x, t) \in D^{+}$, and set $(p, \tau):=\left(\nabla \phi(x, t), \partial_{t} \phi(x, t)\right)$. Then $\phi /(1+\rho(\theta))$ is a test function for $d$ at $(x, t)$. By Remark 1 we see that

$$
\frac{\tau}{1+\rho(\theta)}+H\left(x-d(x, t) \frac{p}{1+\rho(\theta)}, \frac{p}{1+\rho(\theta)}\right) \geqq 0
$$

and that $|p /(1+\rho(\theta))|=1$. Using (5.2) and (5.1), we observe

$$
\begin{aligned}
\tau+H(x, p) & =(1+\rho(\theta))\left\{\frac{\tau}{1+\rho(\theta)}+\frac{1}{1+\rho(\theta)} H(x, p)\right\} \\
& \geqq(1+\rho(\theta))\left\{-H\left(x-d(x, t) \frac{p}{1+\rho(\theta)}, \frac{p}{1+\rho(\theta)}\right)+H\left(x, \frac{p}{1+\rho(\theta)}\right)\right\} \\
& \geqq-(1+\rho(\theta)) L_{1} \min \{3 d(x, t), 2\} \geqq-2 L_{1} \min \{3 d(x, t), 2\} .
\end{aligned}
$$

Since $|p|=1+\rho(\theta)$, we have $-h(p) \geqq g(\rho(\theta))=g\left(\tilde{g}\left(\theta^{-\alpha}\right)\right)=\theta^{-\alpha}$. Also, the monotonicity of $\beta$ gives $\beta(d(x, t)) \leqq \beta\left(v^{\theta}(x, t)\right)$. Summarizing the above, we get

$$
I:=\tau+H(x, p)-\theta \beta\left(v^{\theta}(x, t)\right) h(p) \geqq-2 L_{1} \min \{3 d(x, t), 2\}+\theta^{1-\alpha} \beta(d(x, t)) .
$$

If $d(x, t) \geqq \mu_{1}$, then $I \geqq-2 L_{1} \cdot 2+\theta^{1-\alpha} \beta\left(\mu_{1}\right)$. The right-hand side is non-negative for large $\theta$. In the case where $0<d(x, t) \leqq \mu_{1}$, (B1) implies that

$$
I \geqq-2 L_{1} \cdot 3 d(x, t)+\theta^{1-\alpha} b_{1} d(x, t)=d(x, t)\left\{-6 L_{1}+b_{1} \theta^{1-\alpha}\right\},
$$

and therefore $I \geqq 0$ for $\theta>0$ large. Hence we conclude that $v^{\theta}$ is a supersolution.

The remaining assertion is a consequence of the comparison principle. In fact, it is easy to see that $v_{+}^{\theta}$, the plus part of $v^{\theta}$, is a supersolution of (1.9) in the whole $\mathbf{R}^{n} \times(0, T)$ as is mentioned in [11, 1st paragraph of Section 3.2]. Thus $u^{\theta} \leqq v_{+}^{\theta}$ in $\mathbf{R}^{n} \times(0, T)$, and in particular $0<u^{\theta} \leqq v^{\theta}$ in $D^{+}$. Similarly, considering the minus part of $v^{\theta}$ gives $v^{\theta} \leqq u^{\theta}<0$ in $D^{-}$. On $\Gamma$ we have $u^{\theta}=v^{\theta}$.

Example 4. For our typical $h(p)=1-|p|$, we see that $g(r)=r, \tilde{g}(s)=s$, and so $\rho(\theta)=\tilde{g}\left(\theta^{-\alpha}\right)=\theta^{-\alpha}$. Theorem 5.1 thus yields $\left|u^{\theta}\right| \leqq\left(1+\theta^{-\alpha}\right)|d|$ for any $\alpha \in(0,1)$. 


\section{Acknowledgements}

The author is grateful to Professor Norikazu Yamaguchi for discussions on numerical computations and permission to use the figures in this paper. This work was supported by JSPS KAKENHI Grant-in-Aid for Young Scientists (B) No. 16K17621.

\section{References}

[1] M. Bardi, I. Capuzzo-Dolcetta, Optimal control and viscosity solutions of HamiltonJacobi-Bellman equations, With appendices by Maurizio Falcone and Pierpaolo Soravia, Systems \& Control: Foundations \& Applications, Birkhäuser Boston, Inc., Boston, MA, 1997.

[2] G. Barles, Uniqueness and regularity results for first-order Hamilton-Jacobi equations, Indiana Univ. Math. J. 39 (1990), no. 2, 443-466.

[3] Y.-G. Chen, Y. Giga, S. Goto, Uniqueness and existence of viscosity solutions of generalized mean curvature flow equations, J. Differential Geom. 33 (1991), no. 3, 749-786.

[4] M. G. Crandall, H. Ishii, P. L. Lions, User's guide to viscosity solutions of second order partial differential equations, Bull. Amer. Math. Soc. (N.S.) 27 (1992), no. 1, 1-67.

[5] L. C. Evans, H. M. Soner, P. E. Souganidis, Phase transitions and generalized motion by mean curvature, Comm. Pure Appl. Math. 45 (1992), no. 9, 1097-1123.

[6] L. C. Evans, J. Spruck, Motion of level sets by mean curvature. I, J. Differential Geom. 33 (1991), no. 3, 635-681.

[7] Y. Fujita, Lower estimates of $L^{\infty}$-norm of gradients for Cauchy problems, J. Math. Anal. Appl. 458 (2018), no. 2, 910-924.

[8] Y. Giga, Surface evolution equations: A level set approach, Monographs in Mathematics, 99, Birkhauser Verlag, Basel, 2006.

[9] N. Hamamuki, A few topics related to maximum principles, Ph.D. thesis, The University of Tokyo (2013).

[10] N. Hamamuki, An improved level set method for Hamilton-Jacobi equations, RIMS Kokyuroku, No. 1962 (2015), 27-44. (Japanese)

[11] N. Hamamuki, E. Ntovoris, A rigorous setting for the reinitialization of first order level set equations, Interfaces Free Bound. 18 (2016), no. 4, 579-621.

[12] M. Kimura, Geometry of hypersurfaces and moving hypersurfaces in $\mathbf{R}^{m}$ for the study of moving boundary problems, Topics in mathematical modeling, 39-93, Jindřich Nečas Cent. Math. Model. Lect. Notes, 4, Matfyzpress, Prague, 2008.

[13] O. Ley, Lower-bound gradient estimates for first-order Hamilton-Jacobi equations and applications to the regularity of propagating fronts, Adv. Differential Equations 6 (2001), no. 5, 547-576.

[14] O. Pironneau, Finite element methods for fluids, Translated from the French, John Wiley \& Sons, Ltd., Chichester; Masson, Paris, 1989. 
[15] I. Roisman, Implicit surface method for numerical simulations of moving interfaces, Lecture at the International Workshop on "Transport Processes at Fluidic Interfaces from Experimental to Mathematical Analysis" in Aachen (Germany), December 5-7, 2011.

[16] M. Sussman, E. Fatemi, An efficient, interface-preserving level set redistancing algorithm and its application to interfacial incompressible fluid flow, SIAM J. Sci. Comput. 20 (1999), no. 4, 1165-1191.

[17] M. Sussman, P. Smereka, S. Osher, A level set approach for computing solutions to incompressible two-phase flow, J. Comput. Phys. 114 (1994), no. 1, 146-159. 